PROCEEDINGS OF THE

AMERICAN MATHEMATICAL SOCIETY

Volume 140, Number 11, November 2012, Pages 3693-3701

S 0002-9939(2012)11257-2

Article electronically published on March 2, 2012

\title{
SHORT SUMS OF MULTIPLICATIVE FUNCTIONS
}

\author{
VISHAAL KAPOOR
}

(Communicated by Matthew A. Papanikolas)

Abstract. We show that on a short interval, $x<n \leq x+w$, the average value of a complex-valued multiplicative function $f(n)$ that is sufficiently close to 1 on primes and bounded on prime powers, tends to

$$
C_{f}=\prod_{p}\left(1-\frac{1}{p}\right)\left(1+\frac{f(p)}{p}+\frac{f\left(p^{2}\right)}{p^{2}}+\ldots\right),
$$

provided the interval is sufficiently long with respect to $x$.

The literature is rich with asymptotic formulae for the sum of a multiplicative function over long intervals $n \leq x$. In contrast, little is known about multiplicative functions summed over short intervals $x<n \leq x+y$ except in special circumstances. In 2002, Bordellès showed that under the hypothesis that $f(n)$ is a real-valued multiplicative function taking values in $[0,1]$ and assuming the value 1 on primes, $f(n)$ has an average value tending to

$$
C_{f}=\prod_{p}\left(1-\frac{1}{p}\right)\left(1+\frac{f(p)}{p}+\frac{f\left(p^{2}\right)}{p^{2}}+\ldots\right)
$$

on the short interval provided $y \geq x^{1 / 5+\varepsilon}$ for some $\varepsilon>0$. In this article, we extend Bordellès's theorem to a larger class of functions, lifting the requirement that $f(n)$ be real-valued and weakening the hypothesis on the values of $f(n)$ on the primes. More specifically, we allow any complex-valued multiplicative function that is uniformly bounded on the prime powers and "close" to 1 on the primes.

Theorem 1. Let $A$ and $\theta$ be positive constants with $\theta \geq 4 / 5$. Define $\mathcal{F}_{A, \theta}$ to be the class of complex-valued multiplicative functions $f(n)$ which are bounded by $A$ on prime powers and satisfy the inequality

$$
|f(p)-1| \leq A p^{-\theta}
$$

for all primes $p$. For any $f \in \mathcal{F}_{A, \theta}$ if $\theta \geq 4 / 5$, then

$$
\sum_{x<n \leq x+w} f(n)=w C_{f}+O(\Delta(x, w, \varepsilon))
$$

for all $1 \leq w \leq x$, where

$$
\Delta(x, w, \varepsilon)=\left\{\begin{array}{cl}
x^{1 / 5+\varepsilon}+x^{1 / 15+\varepsilon} w^{2 / 3}+x^{-1 / 10+\varepsilon} w & \text { when } w \leq x^{3 / 5}, \\
x^{1 / 2+\varepsilon} & \text { when } w>x^{3 / 5}
\end{array}\right.
$$

Here $\varepsilon>0$ is sufficiently small, and the implicit constant in the Vinogradov notation depends on $A, \theta$ and $\varepsilon$.

Received by the editors October 6, 2010 and, in revised form, April 21, 2011.

2010 Mathematics Subject Classification. Primary 11N37.

(C)2012 American Mathematical Society Reverts to public domain 28 years from publication 3693 
When $w \leq x^{3 / 5}$, the three expressions $x^{1 / 5+\varepsilon}, x^{1 / 15+\varepsilon} w^{2 / 3}$, and $x^{-1 / 10+\varepsilon} w$ dominate the error term in the ranges $w \leq x^{1 / 5}, x^{1 / 5}<w \leq x^{1 / 2}$, and $w>x^{1 / 2}$ respectively. In particular this theorem is nontrivial when $w \geq x^{1 / 5+\varepsilon}$.

We note that the range of $\theta$ can be enlarged to $\theta>1 / 2$. However, in the range $1 / 2<\theta<4 / 5$ the error term depends heavily on the choice of $\theta$, so we omit the details and refer the reader to the comments following the proof of Theorem 1

For an application of Theorem 11 we find asymptotic formulae for short sums of the functions $n / \varphi(n)$ and $\sigma(n) / n$ over squarefree positive integers $n$. Both of these sums involve functions that are not identically 1 on the set of primes and that have values outside $[0,1]$ and thus cannot be handled using Bordellès's theorem. Applying Theorem 11 with $\theta=1$ gives the short interval asymptotic formulae:

Corollary 2. For $1 \leq w \leq x$ we have both

$$
\sum_{\substack{x<n \leq x+w \\ n \text { squarefree }}} \frac{n}{\varphi(n)}=w+O(\Delta(x, w, \varepsilon))
$$

and

$$
\sum_{\substack{x<n \leq x+w \\ n \text { squarefree }}} \frac{\sigma(n)}{n}=\frac{w}{\zeta(3)}+O(\Delta(x, w, \varepsilon)),
$$

where $\varepsilon>0$. Both of these formulae are nontrivial when $w>x^{1 / 5+\varepsilon}$.

In what follows, we will not state the implicit dependence of $A, \theta$, or $\varepsilon$ in the Vinogradov and big-Oh notation. We use the notation $\lfloor a\rfloor$ to denote the largest integer $\leq a$. The Dirichlet convolution of two arithmetic functions $f$ and $g$ is defined as

$$
(f * g)(n)=\sum_{a b=n} f(a) g(b),
$$

where $a, b$ range over positive integers. Recall that a squarefree number is a natural number not divisible by the square of any prime, and a squarefull number is a natural number with every prime factor appearing with multiplicity at least 2 .

We outline our strategy to prove Theorem 1. We begin by establishing two simple lemmata and stating the more technical Lemma 5, this is followed by the proof of Theorem 1. We will require two results to prove Lemma 5. We then state these results and prove the lemma.

Lemma 3. Let $\varepsilon>0$. For $z \geq 1$ we have

$$
\sum_{\substack{m \leq z \\ m \text { squarefull }}} m^{\varepsilon} \ll z^{1 / 2+\varepsilon}
$$

and we have

$$
\sum_{\substack{m>z \\ m \text { squarefull }}} \frac{1}{m^{1-\varepsilon}} \ll \frac{1}{z^{1 / 2-\varepsilon}}
$$

if $\varepsilon<1 / 2$. 
Proof. We prove the second result; the proof of the first is analogous. Let $S(t)$ be the number of squarefull numbers $\leq t$. Using integration by parts on the RiemannSteltjies integral,

$$
\sum_{\substack{m>z \\ m \text { squarefull }}} \frac{1}{m^{1-\varepsilon}}=\left.\int_{z}^{\infty} \frac{1}{t^{1-\varepsilon}} d S(t) \ll \frac{S(t)}{t^{1-\varepsilon}}\right|_{z} ^{\infty}+\int_{z}^{\infty} \frac{S(t)}{t^{2-\varepsilon}} d t .
$$

Golomb has shown that $S(t) \ll \sqrt{t}$ (see [4), so (4) is $\ll z^{-1 / 2+\varepsilon}$, as required.

Lemma 4. Suppose $f \in \mathcal{F}_{A, \theta}$ for some $A>0$, and $\theta \geq 1 / 2+\varepsilon$, where $\varepsilon>0$. Let $g=f * \mu$. Then for $y \geq 1$ we have

$$
\sum_{d \leq y}|g(d)| \ll y^{1 / 2+\varepsilon}
$$

and we have

$$
\sum_{d>y} \frac{g(d)}{d} \ll y^{\varepsilon-1 / 2}
$$

when $\varepsilon<1 / 2$.

Proof. On primes $p,|g(p)| \leq A / p^{\theta}$ and on higher prime powers $\left|g\left(p^{k}\right)\right| \leq 2 A$. Thus for squarefree $n$, one has

$$
g(n) \ll n^{\varepsilon-\theta}
$$

following from the well-known upper bound $A^{\omega(n)} \ll_{A, \varepsilon} n^{\varepsilon}$. For arbitrary $n$, we have

$$
g(n) \ll n^{\varepsilon} .
$$

We use (5) and (6) to find upper bounds on the sums $\sum_{d<y}|g(d)|$ and $\sum_{d>y} g(d) / d$. One can uniquely parameterize $d \leq y$ as the product of relatively prime $m$ and $n$, with $m$ squarefull and $n$ squarefree. Thus,

$$
\begin{aligned}
\sum_{d \leq y}|g(d)| & =\sum_{\substack{n \leq y \\
n \text { squarefree }}}|g(n)| \sum_{\substack{n m \leq y \\
(m, n)=1 \\
m \text { squarefull }}}|g(m)| \\
& \ll \sum_{\substack{n \leq y \\
n \text { squarefree }}} \frac{1}{n^{\theta-\varepsilon}} \sum_{\substack{m \leq y / n \\
m \text { squarefull }}} m^{\varepsilon} .
\end{aligned}
$$

By (2) this is

$$
\ll \sum_{\substack{n \leq y \\ n \text { squarefree }}} \frac{1}{n^{\theta-\varepsilon}}\left(\frac{y}{n}\right)^{1 / 2+\varepsilon}=y^{1 / 2+\varepsilon} \sum_{n} \frac{1}{n^{\theta+1 / 2}} \ll y^{1 / 2+\varepsilon}
$$

since $\theta>1 / 2$. This establishes the first estimate. 
To deal with the second estimate, we write

$$
\begin{aligned}
\sum_{d>y} \frac{g(d)}{d} & =\sum_{n \text { squarefree }} \frac{g(n)}{n} \sum_{\substack{n m>y \\
(m, n)=1 \\
m \text { squarefull }}} \frac{g(m)}{m} \\
& \ll \sum_{n \text { squarefree }} \frac{1}{n^{\theta+1-\varepsilon}} \sum_{\substack{m \geq y / n \\
m \text { squarefull }}} \frac{1}{m^{1-\varepsilon}} .
\end{aligned}
$$

By (3) this is

$$
\begin{aligned}
& \ll \sum_{n \text { squarefree }} \frac{1}{n^{\theta+1-\varepsilon}}\left(\frac{n}{y}\right)^{1 / 2-\varepsilon} \\
& \ll y^{\varepsilon-1 / 2} \sum_{n} \frac{1}{n^{\theta+1 / 2}} \\
& \ll_{\theta} y^{\varepsilon-1 / 2},
\end{aligned}
$$

when $\varepsilon<1 / 2$. The inner sum converges because $\theta>1 / 2$.

Lemma 5. There exists a sufficiently small positive constant $c_{0}$ such that if $Y \leq$ $c_{0} X^{1 / 2}$ and $X \geq 1 / 2$, then

$$
\sum_{\substack{Y<m \leq X+Y \\ m}}\left(\left\lfloor\frac{X+Y}{m}\right\rfloor-\left\lfloor\frac{X}{m}\right\rfloor\right) \ll X^{\varepsilon} \mathcal{E}(X, Y),
$$

where $\mathcal{E}(X, Y)=X^{1 / 15} Y^{2 / 3}+Y^{4 / 5}+X^{1 / 5}+(X Y)^{1 / 6}$, and $\varepsilon>0$.

Lemma 5 is based on lattice point estimates of Huxley and Sargos (see [5, 6]), and Filaseta and Trifonov [3, Theorem 7]; we summarize these results in Lemma 7 . Lemma 5 is essentially proved by Bordellès over the two papers [1, 2]. For the convenience of the reader, we will collect the details of the proof following the proof of Theorem 1 .

Now, we are ready to prove Theorem 1 Like Bordellès, we express $f(n)$ as the Dirichlet convolution of the identity function and a second function, $g(n)$, which is small in magnitude in lieu of the hypotheses imposed on $f(n)$. However, because we have relaxed the conditions on $f(n)$ at primes, we are left with more technical estimates on $g(n)$. Further, we will consider short intervals of length $\leq x$, where Bordellès considers intervals of length $\ll x^{1 / 2}$. In this wider consideration, we will use the estimate of Filaseta and Trifonov (18) in as large a range as possible and use the estimates of Huxley and Sargos (17) otherwise.

Proof of Theorem 1. Let $g=f * \mu$ and $1 \leq y \leq x$. Write

$$
\begin{aligned}
\sum_{x<n \leq x+y} f(n) & =\sum_{d \leq x+y} g(d)(\lfloor(x+y) / d\rfloor-\lfloor x / d\rfloor) \\
& =\left(\sum_{d \leq y}+\sum_{y<d \leq x+y}\right) g(d)(\lfloor(x+y) / d\rfloor-\lfloor x / d\rfloor) \\
& =\Sigma_{1}+\Sigma_{2} .
\end{aligned}
$$


Then

$$
\Sigma_{1}=y \sum_{d=1}^{\infty} g(d) / d+O\left(y\left|\sum_{d>y} g(d) / d\right|\right)+O\left(\sum_{d \leq y}|g(d)|\right) .
$$

Applying Lemma 4 to the error terms and expressing the infinite sum as an Euler product gives

$$
\Sigma_{1}=y \mathcal{C}_{f}+O\left(y^{1 / 2+\varepsilon}\right)
$$

where $\mathcal{C}_{f}=\prod_{p}(1-1 / p)\left(1+f(p) / p+f\left(p^{2}\right) / p^{2}+\ldots\right)$.

Now we consider $\Sigma_{2}$ :

$$
\begin{aligned}
\left|\Sigma_{2}\right| & \leq \sum_{\substack{n \text { squarefree } \\
n \leq 2 x}}|g(n)| \sum_{\substack{y / n<m \leq(x+y) / n \\
(m, n)=1 \\
m \text { squarefull }}}|g(m)|\left(\left\lfloor\frac{(x+y) / n}{m}\right\rfloor-\left\lfloor\frac{x / n}{m}\right\rfloor\right) \\
& \ll \sum_{n \leq 2 x} \frac{1}{n^{\theta-\varepsilon}}\left(\frac{x+y}{n}\right)^{\varepsilon} \sum_{\substack{y / n<m \leq(x+y) / n \\
m \text { squarefull }}}\left(\left\lfloor\frac{(x+y) / n}{m}\right\rfloor-\left\lfloor\frac{x / n}{m}\right\rfloor\right) .
\end{aligned}
$$

Applying Lemma 5 to the inner sum and noting that $(x+y)^{\varepsilon} \ll x^{\varepsilon}$ for $y \leq x$, we see that

$$
\Sigma_{2} \ll x^{2 \varepsilon} \sum_{n \leq 2 x} \frac{1}{n^{\theta}} \mathcal{E}(x / n, y / n) \ll x^{2 \varepsilon} \mathcal{E}(x, y),
$$

where we have used the requirement that $\theta \geq 4 / 5$ to ensure convergence.

Depending on the size of $w$ in relation to $x$, we will apply (10) and (11) to the entire sum $\sum_{w<n<x+w} f(n)$ or to sums of $f(n)$ over shorter intervals. In what follows, $c_{0}$ is the constant appearing in Lemma 5.

Case $w<c_{0} x^{1 / 2}$ : In this case when $w$ is small enough, we simply choose $y=w$. Then

$$
\begin{aligned}
\sum_{x<n \leq x+w} f(n) & =w \mathcal{C}_{f}+O\left(w^{1 / 2+\varepsilon}+x^{2 \varepsilon}\left(x^{1 / 15} w^{2 / 3}+w^{4 / 5}+x^{1 / 5}+(x w)^{1 / 6}\right)\right) \\
& =w \mathcal{C}_{f}+O\left(x^{1 / 15+2 \varepsilon} w^{2 / 3}+x^{1 / 5+2 \varepsilon}\right)
\end{aligned}
$$

Case $c_{0} x^{1 / 2} \leq w<x^{3 / 5}$ : We choose $y=c_{0} x^{1 / 2}$ and split up our range of summation into blocks of length at most $\lfloor y\rfloor$. We have

$$
\sum_{x<n \leq x+w} f(n)=\sum_{1 \leq k \leq\lfloor w / y\rfloor} \sum_{x+(k-1) y<n \leq x+k y} f(n)+\sum_{x+y\lfloor w / y\rfloor<n \leq x+w} f(n) .
$$

Applying (10) and (11) to each block gives

$$
\begin{aligned}
& w \mathcal{C}_{f}+O\left\{w y^{-1 / 2+\varepsilon}+z^{1 / 2+\varepsilon}+\sum_{1 \leq k \leq\lfloor w / y\rfloor} x^{2 \varepsilon} \mathcal{E}(x+(k-1) y, y)\right. \\
& \left.+x^{2 \varepsilon} \mathcal{E}(x+y\lfloor w / y\rfloor, z)\right\}
\end{aligned}
$$

where $z=w-y\lfloor w / y\rfloor$. 
We note that $z \leq y$, and if $w \leq x$, then both $x+(k-1) y$ and $x+y\lfloor w / y\rfloor$ are $\leq 2 x$. Thus both of the terms $\mathcal{E}(x+(k-1) y, y)$, and $\mathcal{E}(x+y\lfloor w / y\rfloor, z)$ are $\ll \mathcal{E}(x, y) \ll x^{2 / 5}$, plugging in $y=c_{0} x^{1 / 2}$. Thus the error term in (12) is

$$
\ll w y^{-1 / 2+\varepsilon}+y^{1 / 2+\varepsilon}+(w / y+1) x^{2 / 5+2 \varepsilon} \ll x^{-1 / 10+2 \varepsilon} w+x^{2 / 5+2 \varepsilon} \ll x^{-1 / 10+2 \varepsilon} w,
$$

with the last upper bound following from the fact that $w \geq c_{0} x^{1 / 2}$.

Summing the errors obtained in the previous two cases and replacing ' $2 \varepsilon$ ' with ' $\varepsilon$ ' gives the asymptotic formula

$$
\sum_{w<n \leq x+w} f(n)=w \mathcal{C}_{f}+x^{\varepsilon} O\left(x^{1 / 5}+x^{1 / 15} w^{2 / 3}+x^{-1 / 10} w\right),
$$

which we apply when $w \leq x^{3 / 5}$.

Case $w \geq x^{3 / 5}$ : When $w$ is large enough in comparison with $x$, standard convolution arguments applied to the long sums may be effectively used. We have

$$
\begin{aligned}
\sum_{n \leq u} f(n) & =\sum_{k \leq u} g(k)\lfloor u / k\rfloor \\
& =u \sum_{k=1}^{\infty} \frac{g(k)}{k}+O\left(\sum_{k \leq u}|g(k)|+u \sum_{k>u} \frac{g(k)}{k}\right) .
\end{aligned}
$$

Applying the estimates of Lemma 4 gives

$$
\begin{aligned}
\sum_{n \leq u} f(n) & =u \sum_{k=1}^{\infty} \frac{g(k)}{k}+O\left(u^{1 / 2+\varepsilon}\right) \\
& =u C_{f}+O\left(u^{1 / 2+\varepsilon}\right) .
\end{aligned}
$$

Applying this result for $u=x+w$ and $u=x$ and subtracting give

$$
\sum_{x \leq n \leq x+w} f(n)=w C_{f}+O\left(x^{1 / 2+\varepsilon}\right),
$$

as required.

In Theorem 1, although one requires $\theta \geq 4 / 5$ for the bound on $\Sigma_{2}$ in equation (11), we remark that some mileage may be obtained in the range $1 / 2<\theta<4 / 5$. In this range of $\theta$, one may sum the series involving $n$ up to $x$ to obtain the upper bound

$$
\Sigma_{2} \ll x^{3 \varepsilon} \mathcal{E}_{\theta}^{\prime}(x, y)+x^{2 \varepsilon} \mathcal{E}(x, y)
$$

where

$$
\mathcal{E}_{\theta}^{\prime}(X, Y)=X^{1 / 3-\theta} Y^{2 / 3}+X^{11 / 15-\theta} Y^{4 / 15}+X^{1-\theta}+X^{5 / 6-\theta} Y^{1 / 6} .
$$

Here we have used the bound $\sum_{n \leq u} 1 / n^{a} \ll 1+u^{1+\varepsilon-a}$ valid for $a>0$ and including a $u^{\varepsilon}$ to bound $\log u$ in the case $a=1$. One may then proceed similarly as in the proof of Theorem 1 again using standard convolution arguments applied to the long sums when appropriate. We omit the details as they depend on the choice of $\theta$. 
The proof of Lemma 5 requires the following:

Lemma 6. For $1 \leq y \leq x$, and $r$ a positive integer, one has

$$
\mathcal{T}(x, y ; r):=\sum_{x<c^{r} \leq x+y}\left(\left\lfloor\frac{x+y}{c^{r}}\right\rfloor-\left\lfloor\frac{x}{c^{r}}\right\rfloor\right) \ll x^{\varepsilon / r}(\lfloor x+y\rfloor-\lfloor x\rfloor) .
$$

One may prove this lemma by expressing $\mathcal{T}(x, y ; r)$ as

$$
\sum_{x<m \leq x+y} \sum_{\substack{x<c^{r} \leq x+y \\ c^{r} \mid m}} 1 \leq \sum_{x<m \leq x+y} \sum_{c^{r} \mid m} 1 \ll_{\varepsilon} x^{\varepsilon / r} \sum_{x<m \leq x+y} 1,
$$

using the fact that $\sum_{c^{r} \mid m} 1 \ll_{\varepsilon} m^{\varepsilon / r}$.

Let $\varphi$ be a real-valued function on the real numbers, and let $\delta>0$. We define

$$
\mathcal{R}(\varphi, N, \delta)=\mid\{n \in \mathbb{Z}: N<n \leq 2 N \text { and } \exists m \in \mathbb{Z} \text { s.t. }|\varphi(n)-m| \leq \delta\} \mid .
$$

Then for a function $\gamma(n)>0$ with $\delta=\delta(N)=\max _{N<n \leq 2 N} \gamma(n)$ one has

$$
\sum_{n \leq M}(\lfloor\varphi(n)+\gamma(n)\rfloor-\lfloor\varphi(n)\rfloor) \ll \max _{N \leq M} \mathcal{R}(\varphi, N, \delta) \log M
$$

when $M \geq 2$. We shall use this fact along with the following upper bounds on $\mathcal{R}(\varphi, N, \delta)$ that can be found in [1].

Lemma 7. Let $\varphi \in C^{k}(N, 2 N]$ be a real-valued function for some $k \geq 2$, and let $\delta>0$. If there exists $\lambda_{k}=\lambda_{k}(N)$ such that $\left|\varphi^{(k)}(x)\right| \asymp \lambda_{k}$ when $N<x \leq 2 N$, then

$$
\mathcal{R}(\varphi, N, \delta) \ll N \lambda_{k}^{2 / k(k+1)}+N \delta^{2 / k(k-1)}+\left(\delta \lambda_{k}^{-1}\right)^{1 / k}+1 .
$$

There exists a sufficiently small positive constant $c_{0}=c_{0}(k)$ such that if $N^{k-1} \delta \leq c_{0}$ and $N \leq x^{1 / k}$, then

$$
\mathcal{R}\left(x / n^{k}, N, \delta\right) \ll_{k} x^{1 /(2 k+1)}+x^{1 /(6 k+3)} \delta N^{\left(6 k^{2}+k-1\right) /(6 k+3)} .
$$

Proof of Lemma 5. We sketch the proof from "On Short Sums of Certain Multiplicative Functions" [1] and "Corrigendum to On Short Sums of Certain Multiplicative Functions" [2].

The sum over squarefull $m$ in (8) can be parameterized in terms of $a, b$ writing $m=a^{2} b^{3}$ with $b$ squarefree. Since $m>Y$, one of $a$ and $b$ is greater than $Y^{1 / 5}$. Thus

$$
\begin{aligned}
& \sum_{\substack{Y<m \leq X+Y \\
m \text { squarefull }}}\left(\left\lfloor\frac{X+Y}{m}\right\rfloor-\left\lfloor\frac{X}{m}\right\rfloor\right) \\
& \ll \sum_{Y^{1 / 5}<a \leq(X+Y)^{1 / 2}} \mathcal{T}\left(X / a^{2}, Y / a^{2} ; 3\right)+\sum_{Y^{1 / 5}<b \leq(X+Y)^{1 / 3}} \mathcal{T}\left(X / b^{3}, Y / b^{3} ; 2\right) .
\end{aligned}
$$

Lemma 6 gives us the upper bound

$$
\begin{aligned}
X^{\varepsilon / 3} \sum_{Y^{1 / 5}<a \leq(X+Y)^{1 / 2}}\left(\left\lfloor\frac{X+Y}{a^{2}}\right\rfloor\right. & \left.-\left\lfloor\frac{X}{a^{2}}\right\rfloor\right) \\
& +X^{\varepsilon / 2} \sum_{Y^{1 / 5}<b \leq(X+Y)^{1 / 3}}\left(\left\lfloor\frac{X+Y}{b^{3}}\right\rfloor-\left\lfloor\frac{X}{b^{3}}\right\rfloor\right) .
\end{aligned}
$$


The trivial estimates

$$
\left\lfloor(X+Y) / a^{2}\right\rfloor-\left\lfloor X / a^{2}\right\rfloor \leq Y / a^{2}
$$

and

$$
\left\lfloor(X+Y) / b^{3}\right\rfloor-\left\lfloor X / b^{3}\right\rfloor \leq Y / b^{3}
$$

applied for the ranges $Y^{1 / 5}<a \leq(2 Y)^{1 / 2}$ and $Y^{1 / 5}<b \leq(2 Y)^{1 / 3}$ respectively give an upper bound of $X^{\varepsilon / 2} Y^{4 / 5}$.

By equation (16) we see that

$$
\sum_{(2 Y)^{1 / 3}<b \leq(X+Y)^{1 / 3}}\left(\left\lfloor\frac{X+Y}{b^{3}}\right\rfloor-\left\lfloor\frac{X}{b^{3}}\right\rfloor\right) \ll \max _{B \leq(X+Y)^{1 / 3}} \mathcal{R}\left(\frac{X}{b^{3}}, B, \frac{Y}{B^{3}}\right) \log X .
$$

Equation (17) of Lemma 7 with $k=3$ and $\lambda_{3}(B)=X B^{-6}$ gives

$$
X^{\varepsilon / 2} \sum_{(2 Y)^{1 / 3}<b \leq(X+Y)^{1 / 3}}\left(\left\lfloor\frac{X+Y}{b^{3}}\right\rfloor-\left\lfloor\frac{X}{b^{3}}\right\rfloor\right) \ll X^{\varepsilon}\left(X^{1 / 6}+Y^{1 / 3}\right) .
$$

Applying equations (17) and (16) similarly to $\varphi(a)=X / a^{2}$ with $k=3, \lambda_{3}=X A^{-5}$, and $\delta=Y A^{-2}$ gives

$$
X^{\varepsilon / 3} \sum_{(2 Y)^{1 / 2}<a \leq c_{0}^{-1} Y}\left(\left\lfloor\frac{X+Y}{a^{2}}\right\rfloor-\left\lfloor\frac{X}{a^{2}}\right\rfloor\right) \ll X^{\varepsilon}\left((X Y)^{1 / 6}+Y^{2 / 3}+Y^{4 / 3} X^{-1 / 3}\right) .
$$

Equation (18) of Lemma 7 with (16) gives the bound

$$
X^{\varepsilon / 3} \sum_{c_{0}^{-1} Y<a \leq(X+Y)^{1 / 2}}\left(\left\lfloor\frac{X+Y}{a^{2}}\right\rfloor-\left\lfloor\frac{X}{a^{2}}\right\rfloor\right) \ll X^{\varepsilon}\left(X^{1 / 5}+X^{1 / 15} Y^{2 / 3}\right) .
$$

Here we have chosen the cutoff $c_{0}^{-1} Y$ so that we may apply (18) of Lemma 7 but doing so has introduced the requirement that $(X+Y)^{1 / 2} \geq c_{0}^{-1} Y$, which is satisfied when $Y \leq c_{0} X^{1 / 2}$.

Summing the estimates gives the upper bound

$$
O\left(X^{\varepsilon}\left(Y^{4 / 5}+X^{1 / 5}+X^{1 / 15} Y^{2 / 3}+(X Y)^{1 / 6}\right)\right) .
$$

\section{ACKNOWLEDGEMENTS}

The author is grateful for the limitless patience and sound advice of his supervisor, Greg Martin. He would like to thank the anonymous referee for providing very detailed and helpful feedback on the exposition and for suggesting the method to enlarge the range of $\theta$. He would like to thank Kevin Ford for suggesting the examples in Corollary 2 .

\section{REFERENCES}

1. Olivier Bordellès, On short sums of certain multiplicative functions, JIPAM, Journal of Inequalities in Pure and Applied Mathematics 3 (2002), no. 5, Article 70, 6 pp. (electronic). MR1966505 (2004a:11097)

2. __ Corrigendum to: "On short sums of certain multiplicative functions", JIPAM, Journal of Inequalities in Pure and Applied Mathematics 5 (2004), no. 3, Article 81, 3 pp. (electronic). MR2084890 (2005d:11141)

3. Michael Filaseta and Ognian Trifonov, The distribution of fractional parts with applications to gap results in number theory, Proc. London Math. Soc. (3) 73 (1996), no. 2, 241-278. MR.1397690 (2000i:11110) 
4. S. W. Golomb, Powerful numbers, Amer. Math. Monthly 77 (1970), 848-855. MR0266878 $(42: 1780)$

5. M. N. Huxley and P. Sargos, Points entiers au voisinage d'une courbe plane de classe $C^{n}$, Acta Arith. 69 (1995), no. 4, 359-366. MR1318755 (96a:11065)

6. Martin N. Huxley and Patrick Sargos, Points entiers au voisinage d'une courbe plane de classe $C^{n}$. II, Funct. Approx. Comment. Math. 35 (2006), 91-115. MR.2271609 (2007m:11092)

Google, 1600 Amphitheatre Parkway, Mountain View, California 94043 